\title{
Discouragement Towards Seeking Health Care of Older People in Rural China: The Influence of Culture and Structural Constraints
}

\author{
Xiang Zou
}

\subsection{Introduction}

Within contemporary bioethical writing, ethical deliberations about patients' healthseeking behaviours have long been centred on issues of patients' rights, agency and autonomy. This right-based ethical paradigm, however, is incomplete, as it conveys by and large the values of those privileged patients in developed social settings-i.e. those who tend to be autonomous, who have relatively better access to quality medical care resources and who are able to navigate institutional structures proactively. In contrast, values regarding the health-seeking behaviours of patients in underdeveloped sociocultural contexts have long been omitted.

This chapter speaks to the values of and perspectives towards seeking health care among older patients in Chinese rural settings. Those concerned are in general financially disadvantaged, highly dependent and hence less autonomous and lack proper access to health care. This chapter portrays the discouraged, despairing attitudes about the elderly seeking health care in rural China, as internalised by older patients themselves and expressed by their families and the public. Underlying this discouragement is a widespread cultural value, namely the social expectation that people should endure suffering and hence that the importance of seeking elderly health care should be downplayed. Additionally, this discouraged attitude mirrors the lack of proper access to commodified health care of rural patients as an aspect of the rural-urban structural divide.

Drawing upon empirical data collected from 6 months of field work in a Chinese rural hospital, Qincun Hospital, in 2016, this chapter illustrates the discouraged attitude towards older patients seeking health care through the story of Aunt Chen, an older mother who was hospitalized in Qincun Hospital during the time of the current study, to obtain hospital care and the reactions of her family. Oral informed

X. Zou $(\bowtie)$

Department of Medical Humanities, Southeast University, Nanjing, China

e-mail:xiang.zou@hotmail.com 
consent was given by the family involved in the case study published below. To protect the confidentiality of the research participants, all identifying information was removed. For example, the names used in this chapter-including those of interviewees and locations_-are pseudonyms. The Human Ethics Committee of the University of Otago in New Zealand approved the research (Reference No. 15/106).

\subsection{Aunt Chen's Story}

Aunt Chen, an 83-year-old widow, suffered from multiple chronic symptoms. Over the past decade she had been heavily reliant on inpatient treatment for pain relief. Like most rural residents over 60 years of age in China, Aunt Chen did not have any formal source of income to secure medical care. Given this situation, the responsibility for her long-term care and treatment-related decision-making fell on her family-that is, her three children. Additionally, due to the lack of access to quality health care resources (most of which were located in urban settings), the Qincun Hospital was sought as the most affordable solution to her health care needs.

Over the past decade of health seeking, Aunt Chen's symptoms had continued to progress with much of her care and treatment proving futile. Her family thus felt discouraged and became reluctant to continue support for her medical care. Discouragement was further exacerbated by the fact that her three children had migrated to city areas for employment. Aunt Chen however remained proactive about seeking hospital care and treatment despite these discouragements. The family process of negotiating medical care and family support led to severe conflicts between the two generations. For example, during the current study, there was an occasion that Aunt Chen suffered from a recurrence of coronary heart problems and requested her children to send her to Qincun Hospital. Her family, however, rejected her request since, at that time, the whole family was involved in the preparations for a young family member's wedding ceremony, asserting that nobody had the time or energy to accompany her on a hospital visit. Seeing there was no way out, Aunt Chen threatened to commit suicide during the wedding if her family still rejected her request for a hospital visit: "Don't be busy with the wedding, prepare for my funeral!"

Aunt Chen's threat worked and she was sent over to the hospital where medical drips were promptly offered. The threat, on the other hand, heightened her children's annoyance. For example, Aunt Chen's third son once made a comment about her persistent health seeking and preoccupation with longevity-he saw this as "unreasonable" and said it was "because she was too scared of death". "If you don't [bring her to the hospital], she threatens she will die or will tell other people we children are not filial... Which older person does not have some pain? Does it really need hospital treatment? She had seen her 83rd already. Why can't she just let it go?" The moral concern of being accused as "un-filial" pressure the family to continue their support with Aunt Chen's hospital care and medications.

This view was shared by other people in their circle who agreed that, as an older person, Aunt Chen had asked "too much", and that it was wrong of her to pursue health care and longevity. For instance, Aunt Wang, another 80-year-old mother 
who was also hospitalised, narrated her own experiences and encouraged Aunt Chen to be "reasonable" in the face of family neglect: "Every time I was sick, my children just pretended that they had no idea about it. But that's all right. Being an older person, you need to be content [zhizu] with what you've got!" The view that Aunt Chen was in the wrong was also expressed by a doctor from Qincun Hospital, who said: "You know she [Aunt Chen] will never to be able to get well. It is unwise to be always clinging to life and devote much to medical care...At her age, it is not worth treating that seriously..."

Aunt Chen was just in her 50s when her husband died. To raise her four children, she endured enormous hardships and suffering working as a farm labourer. These hardships and suffering, she believed, were the cause of her ill condition, "I worked too hard, that's why I became so sick when I got old"- an interpretation morally justified her chronic conditions that deserved proper care and support from her children in repayment. Her family's negligence and public accusations made her feel wounded. She resisted them by revealing a strong will to live: "everyone said I should come back home and wait for death. But I'm not prepared for it yet!"

On the other hand, Aunt Chen also internalised the public discouragements, expressing a deep concern of being accused as "greedy" and her family's reluctance to take on the heavy burden of support for her long-term health care: "I know they were so fed up with me. If I continue living in the hospital, they will get very angry and stop offering me anything..."

The result was that Aunt Chen managed to cut short the length of her inpatient stay. After only a few days of treatment, she requested to be discharged and to return home for rehabilitation. It was unknown how she would be treated at home.

\subsection{The Values Arising in This Case}

Aunt Chen's story highlights the prevailing discouraging attitude towards seeking elderly health care in a Chinese rural locality. This discouraging attitude was expressed by Aunt Chen's family and came to be particularly prominent when the family prioritised the younger member's wedding ceremony over seeking for Aunt Chen hospital care and treatment. Discouragement is also revealed in the attitudes of local people who accused Aunt Chen of being immoral for asking "too much" in the way of medical care. Underpinning these discouragements is the value that older people's health was "unworthy of care and treatment" and therefore their proactive health-seeking behaviours are morally "inappropriate" and culturally unacceptable. Instead, what is "appropriate" for an older people is to be self-restrained with seeking health care, being good at enduring chronic pain and suffering, and to consciously reduce the burden of health care on their families. This local perception is in direct opposition to the conventional (in Western medical ethics) positive understanding of "ideal" patients who proactively engage in health-seeking and pursuing personal well-being.

A set of contradictory values arises from her attempts to sustain medical care and family support; however, her struggle with unfavourable values and conventions 
discourages her from doing so. As a patient, Aunt Chen revealed a strong will in pursuing her desire for personal health and longevity. As an older family member, on the other hand, she also bore the moral expectation of being self-restrained and reasonable, prioritising the interests of the whole family and reducing the burden of medical care on them. Torn between these contradictory values, her case provides a lens on what really mattered for Aunt Chen, as a patient or a socially accepted older person, when accommodating these different values and priorities and seeking a middle-way out. It also encourages us to ask what kind of social, cultural and structural backdrop shapes the circumstances that discourages and devalues health care seeking for older people. It is to this that I turn next.

\subsection{The Social, Cultural and Structural Backdrop of Aunt Chen's Story}

The public discouragement illustrated by Aunt Chen's story mirrors cultural values common in many rural regions, that view enduring pain and suffering as being morally "upright" personal virtues. As reported in contemporary literature, personal characteristics such as maintaining thrifty and self-constrained lifestyles, and being good at enduring hardships and conscious of reducing their adult children's financial burdens, are usually perceived as morally upright and positive for older people in rural China [1-3]. These cultural values encourage older people to be selfrestrained with seeking health and much needed care for themselves since they view these as the behaviours "appropriate" for older people and wish to become socially recognised virtuous members of their society.

Related to these cultural values, a discourse of "worthlessness" and ageist discriminations, constructed under the influence of a market economy, also contribute to the devalued significance of care for the aged in China. In the traditional Chinese society, most older people enjoyed a high gerontological status and were honoured for their mastery of wisdom and representation of high moral standards of the society [4]. Throughout the economic reforms of the past three decades, marketization and privatisation have created a new image of self-actualising and self-reliant individuals, under which people were encouraged to bear their own responsibilities for health care and late-age support. Just as the market economy prioritised independence and competitiveness, so it also problematized the image of Aunt Chen, and other older people who like her were frail, feeble and sick, relying on external care and support to survive. Older people had seen a decreased gerontological status from experiencing socioeconomic transformations in China, and their health needs were perceived as a "worthless" socioeconomic burden. Both the state and individual families become reluctant to support aged care. Unwillingness to care is heightened by the massive outwards migration of rural populations to urban areas to seek employment, from which rural families experienced comprised capacities and resources to assist health-seeking for their older members.

Another social force that contributes to the discouragement of health seeking behaviour among the elderly is the lack of access to health care resources that has 
been a product of the persistent rural-urban divide. The Chinese household registration policy (hukou system) [5, 6] designated all Chinese residents to one of two types of households, either rural or urban, based on the types of for the aged employment they were entitled. Based on this divide, rural residents were granted with inferior access to social welfare, such as insurance coverage and social pension systems (in contrast to urban dwellers). The lack of institutional support means rural older residents had to rely on their families to secure health care and late-age support constrained as they were by structural obstacles. Additionally, the hukou system also constructs a sense of inferiority amongst rural residents that by labelling them as "second-class", "backward peasants" $[1,7]$. This social discourse is highly discriminated and further discourages health-seeking of rural older people.

Discouragement to seeking support in old age was further intensified by a lack of trust in the commodified health care industry in China. Since China started market economy reforms in the 1980s, the government gradually withdrew its funding from social welfare systems. The public hospitals became underfunded, and health provides increasingly reliant on revenues generated by fees for high-tech interventions and (often excessive) prescriptions [8]. Meanwhile, as much for the aged of expenditures were devoted to the development of larger hospitals in urban areas, rural hospitals suffered from severe staff and compromised quality of medical care facilities [9]. These institutional changes resulted in heightened financial barriers of accessing hospital care that further disadvantaged rural older people who were of lower socioeconomic status. Discouragement to support elderly health care was further heightened by the public pessimism about the curability and recovery of older people's chronic symptoms, making the public refusal and the perception of elderly health-seeking as a waste of resources culturally legitimated.

\subsection{Conclusions}

Drawing upon the case of health-seeking of Aunt Chen's family collected from a 6-month field work in a rural primary hospital, this chapter portrays a discouraged, dispiriting attitude towards health-seeking of rural older people in China. Underpinning this discouragement is the prevalence of discriminatory attitudes that by downplaying the significance of elderly health care as "worthless" socioeconomic burden. The construction of this "worthless" perception is not only culturerelated but also structure-induced. The cultural belief is that morally prioritising older people's good endurance of pain and suffering and self-restrained personal characteristics significantly contributes to the public discouragements towards elderly health-seeking. In addition, the structural reality of rural-urban divide and socioeconomic deprivations compounds the public discouragements and devaluation. In this respect, this discouraged attitude not only reflects the influence of cultural values but also suggests rural residents' dissatisfaction with the state-based social welfare system in contemporary rural China.

Acknowledgements The author is in debt to all of the participants in the study. 


\subsection{Guide to Further Sources}

1. The Chinese term zhizu literately can be translated as "the feeling of being content" in English. In the Chinese social context, zhizu indicates a disposition that encourages people to be self-restrained with and actively low their expectations on pursuing personal interests and desires for achieving the ends of happiness and welfare. Keeping zhizu has been perceived as a type of personal virtue and thus morally upright and culturally encouraged.

2. For more details about the geographic information of the field site, Chengcun Town (程 村 镇), where the Qingcun Hospital was located, see: https://baike. baidu.com/item/ (in Chinese), access on 15th November, 2019.

\section{References}

1. Long Y, Li LW. "How would we deserve better?" Rural-urban dichotomy in health seeking for the chronically ill elderly in China. Qual Health Res. 2016;26(12):1689-704.

2. Lora-Wainwright A. 'If you can walk and eat, you don't go to hospital': the quest for healthcare in rural Sichuan. In: Duckett J, editor. China's changing welfare mix local perspectives. 1st ed. New York: Taylor and Francis; 2011.

3. Lora-Wainwright A. Fighting for breath: living morally and dying of cancer in a Chinese village. Honolulu: University of Hawaii Press; 2013.

4. Benjamin D, Brandt L, Rozelle S. Aging, wellbeing, and social security in rural northern China. Popul Dev Rev. 2000;26:89-116.

5. Chan KW, Zhang L. The Hukou system and rural-urban migration in China: processes and changes. China Q. 1999;160:818-48.

6. Zhang Z, Treiman DJ. Social origins, hukou conversion, and the wellbeing of urban residents in contemporary China. Soc Sci Res. 2013;42(1):71-89.

7. Zhang L, Ong A. Privatizing China: socialism from afar. Ithaca: Cornell University Press; 2008.

8. Yip WC, Hsiao WC, Chen W, Hu S, Ma J, Maynard A. Early appraisal of China's huge and complex health-care reforms. Lancet. 2012;379(9818):833-42.

9. Zou X, Nie J-B. Access to care by older rural people in a post-reform Chinese hospital: an ethical evaluation of anthropological findings. Asian Bioeth Rev. 2019;11(1):57-68.

Open Access This chapter is licensed under the terms of the Creative Commons Attribution 4.0 International License (http://creativecommons.org/licenses/by/4.0/), which permits use, sharing, adaptation, distribution and reproduction in any medium or format, as long as you give appropriate credit to the original author(s) and the source, provide a link to the Creative Commons license and indicate if changes were made.

The images or other third party material in this chapter are included in the chapter's Creative Commons license, unless indicated otherwise in a credit line to the material. If material is not included in the chapter's Creative Commons license and your intended use is not permitted by statutory regulation or exceeds the permitted use, you will need to obtain permission directly from the copyright holder.

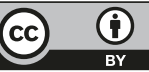

